\title{
Interplay of Couplings between Antiferrodistortive, Ferroelectric, and Strain Degrees of Freedom in Monodomain $\mathrm{PbTiO}_{3} / \mathrm{SrTiO}_{3}$ Superlattices
}

\author{
Pablo Aguado-Puente, Pablo García-Fernández, and Javier Junquera \\ Departamento de Ciencias de la Tierra y Física de la Materia Condensada, Universidad de Cantabria, \\ Cantabria Campus Internacional, Avenida de los Castros s/n, 39005 Santander, Spain
}

(Received 4 May 2011; published 15 November 2011)

\begin{abstract}
We report first-principles calculations on the coupling between epitaxial strain, polarization, and oxygen octahedra rotations in monodomain $\left(\mathrm{PbTiO}_{3}\right)_{n} /\left(\mathrm{SrTiO}_{3}\right)_{n}$ superlattices. We show how the interplay between (i) the epitaxial strain and (ii) the electrostatic conditions can be used to control the orientation of the main axis of the system. The electrostatic constrains at the interface facilitate the polarization rotation and, as a consequence, we predict large piezoelectric responses at epitaxial strains smaller than those required considering only strain effects. In addition, ferroelectric (FE) and antiferrodistortive (AFD) modes are strongly coupled. Usual steric arguments cannot explain this coupling and a covalent model is proposed to account for it. The energy gain due to the FE-AFD coupling decreases with the periodicity of the superlattice, becoming negligible for $n \geq 3$.
\end{abstract}

Superlattices composed of thin layers of ferroelectric and paraelectric (or incipient ferroelectric) $\mathrm{ABO}_{3}$ perovskite oxides have attracted a lot of interest during the past few years [1-3]. The fascination for these layered systems comes from the fact that the properties of epitaxial structures, made by stacking different perovskites, are not a simple combination of the properties of the constituent materials, but exotic phenomena might emerge that fully rely on interfacial effects.

$A B \mathrm{O}_{3}$ perovskite oxides present different phase transition sequences and ground states (GS) involving, among others, zone-center ferroelectric (FE) distortions, characterized by the opposite motion of the cations with respect to the $\mathrm{O}$ cage, and nonpolar zone-boundary antiferrodistortive (AFD) modes, which consist on rotation and tilting of the oxygen octahedra surrounding the $B$ cation [4]. But polar and nonpolar instabilities often compete and tend to suppress each other, so one of these distortions dominates over the others and is the only one that appears in the GS structure. However, the balance is extremely delicate and can be tuned by external electrical and strain [5] fields, or by changing the chemical environment through the use of different materials and periodicities in the stack [6].

One of the most studied systems in the recent literature, the $\left(\mathrm{PbTiO}_{3}\right)_{m} /\left(\mathrm{SrTiO}_{3}\right)_{n}(\mathrm{PTO} / \mathrm{STO})$ superlattice, constitutes a good example where the balance between different instabilities has been observed to be strongly tunable. It was theoretically predicted and experimentally observed that the polarization, tetragonality, and phase transition temperature of the system can be monitored with the number of PTO layers $m$, decreasing monotonically when the PTO volume fraction is reduced $[7,8]$. However, in the limit of ultrashort periods, PTO/STO superlattices exhibit an unexpected recovery of ferroelec- tricity that cannot be accounted for by simple electrostatic considerations alone [9]. In this milestone work, the GS of the system was described as not purely ferroelectric, but involving a trilinear coupling term between two AFD modes, that correspond to in-phase $\left(\mathrm{AFD}_{z i}\right)$ and out-ofphase $\left(\mathrm{AFD}_{z o}\right)$ rotations of the oxygen octahedra around the $z$ axis, that induce a polar $\mathrm{FE}$ distortion $\left(\mathrm{P}_{z}\right)$ in a way compatible with hybrid improper ferroelectricity.

Despite all the previous efforts, two problems remain virtually unexplored. First, although many works have dealt independently with the FE-strain and AFD-strain couplings (see Refs. [5,10], respectively), the influence of the direction and magnitude of local polarization on the rotation of the oxygen octahedra has not received the same attention. The control over the bond angles through the polarization orientation and magnitude could open new routes to generate or enhance magnetoelectric couplings [11]. Second, first-principles simulations addressing the transition between the improper and normal ferroelectric regimes as a function of the periodicity of the superlattice are, to our knowledge, missing in the literature.

In this Letter we theoretically predict large mixed FE-AFD-strain couplings in monodomain PTO/STO superlattices. As a result of these interplays, the phase diagram is much richer than originally assumed [9], with rotation of the polarization away from the superlattice normal, and a strong coupling of the AFD modes with the magnitude and direction of the FE mode. We study the physical origin of this phase diagram and the energetics of the FE and AFD contributions and their coupling with increasing periodicity.

For this study we perform first-principles simulations of $(\mathrm{PTO})_{n} /(\mathrm{STO})_{n}$ superlattices, within the local density approximation (LDA) using the SIESTA code [12]. Very accurate computations are required since the relevant 
differences in energies are 7 orders of magnitude smaller than the absolute value of the energy. Real space integrations are computed in a uniform grid, with an equivalent plane-wave cutoff of 1200 Ry. For the Brillouin zone integrations we use a Monkhorst-Pack sampling equivalent to $12 \times 12 \times 12$ in a five atom perovskite unit cell. Details on the norm-conserving pseudopotentials and the basis set used can be found in Ref. [13]. The superlattices are simulated by means of a supercell approach, where we repeat periodically in space a basic unit cell, that is built stacking alternating $n$-unit-cells-thick layers of PTO and STO along the [001] direction for a global periodicity of $(n / n)$ [Fig. 1(a)]. This structure leads to naturally classify the $\mathrm{TiO}_{6}$ octahedra into four different types (PTO, STO, $\mathrm{P}^{+}$, and $\mathrm{P}^{-}$) depending on the top or bottom $A \mathrm{O}$ layer and the direction of polarization [see Fig. 1(a)]. In-plane lattice vectors are doubled to account for the condensation of AFD instabilities. With the $(2 \times 2)$ in-plane periodicity, $\mathrm{TiO}_{6}$ octahedra are allowed both to rotate an angle $\phi$ around the $z$ axis or to tilt an angle $\theta$ around an axis contained in the $(x, y)$ plane [Fig. 1(b)]. The mechanical boundary conditions imposed by the substrate are implicitly treated by fixing the in-plane lattice constant $a_{\|}$. The use of periodic boundary conditions imposes short-circuit electrical conditions across the whole unit cell.

For every $a_{\|}$, a reference nonpolar structure is then obtained after a constrained relaxation within the $P 4 / \mathrm{mbm}$ group, until the maximum force and $z z$ stress tensor components fall below $0.01 \mathrm{eV} / \AA$ and $0.0001 \mathrm{eV} / \AA^{3}$, respectively. Then, symmetry is broken, displacing coherently the cations by hand, and a second relaxation is carried out without any imposed symmetry.

For the $(2 / 2)$ superlattice we have performed structural relaxations under different in-plane strains, while keeping the global tetragonal symmetry. The misfit strain is defined

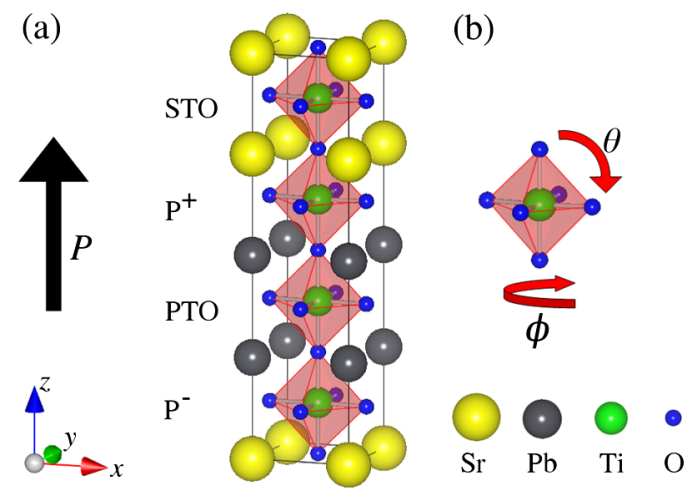

FIG. 1 (color online). (a) Schematic representation of a $(2 / 2)$ PTO/STO superlattice. $\mathrm{TiO}_{6}$ octahedra are labeled according to the chemical identity of the first two neighbor $A O$ planes and the direction of the polarization. (b) Definition of the angles of rotation around the $z$ axis, $\phi$, and tilting around an axis in the $(x, y)$ plane $\theta$ of octahedra. as $\varepsilon=\frac{a_{\|}-a_{0}}{a_{0}}$, where $a_{0}$ is our LDA theoretical lattice constant of cubic bulk STO (3.874 $\AA$ ).

The dependence of the polarization (inferred from the bulk Born effective charges and the local atomic displacements) and the oxygen octahedra rotations and tiltings with the epitaxial strain can be seen in Fig. 2. The first conclusion that can be drawn is the existence of a strong coupling between FE distortions and AFD modes with strain. Both the magnitude and the relevant directions (of the FE polarization and/or the rotation axis of oxygen octahedra) can be tuned by the amount of epitaxial strain.

For large compressive strains the polarization along the $z$ direction ( $c$ phase in Refs. $[14,15]$ ) and the rotations of oxygen octahedra with respect to the same $z$ axis are stabilized, while the tiltings are suppressed. In addition to the effect of the epitaxial strain, the presence of the interfaces plays a twofold role, as can be deduced by comparing the superlattice and bulk results for pure PTO and STO in Fig. 2. (i) The magnitude of the polarization along $z$ is homogeneous throughout the heterostructure. The polarization mismatch at the interface is always

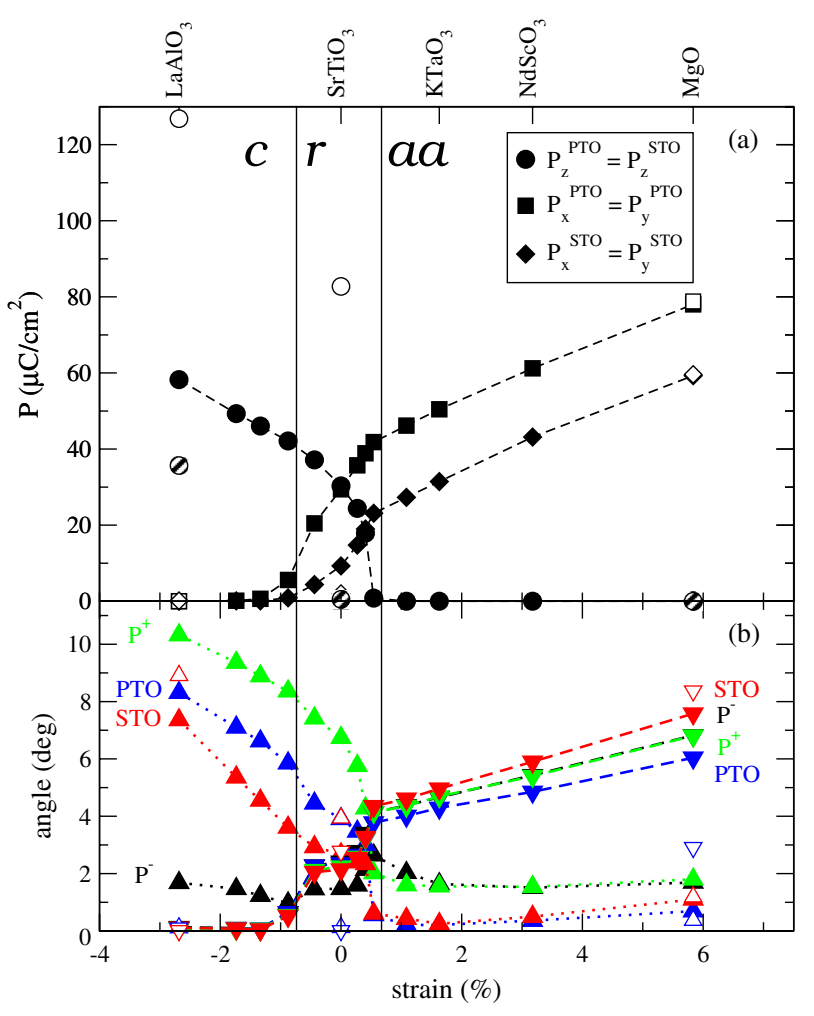

FIG. 2 (color online). (a) Polarization and (b) absolute value of the oxygen octahedra rotations and tiltings in monodomain $(2 / 2)$ superlattices under different epitaxial strains. In (a), empty (patterned) circles correspond to bulk PTO (STO) under the same strain conditions. In (b), the rotation $\phi$ and tilting $\theta$ angles of the $\mathrm{O}$ octahedra (labeled as in Fig. 1) are represented as uppointing triangles and down-pointing triangles, respectively. As in (a), empty symbols refer to bulk values under strain. Top labels indicate strains induced by common substrates. 
smaller than $0.5 \mu \mathrm{C} / \mathrm{cm}^{2}$, highlighting the large electrostatic cost of a polarization discontinuity between the layers $[7,16]$. The price to pay for poling the STO layer is a reduction in the polarization of PTO with respect to bulk [compare open and filled circles in Fig. 2(a)]. (ii) The magnitude of the angle of rotation is different for the different octahedra, a fact that points out a special coupling between the AFD modes and the FE polarization. It is remarkable that, although bulk PTO does not exhibit rotation of oxygen octahedra, the $\mathrm{TiO}_{6}$ octahedra in the superlattice with PTO-like environment inherits part of the AFD character of STO and are forced to rotate.

In the opposite limit, for large tensile strains, the polarization in the most stable configuration lies in the plane, along the [110] direction ( $a a$ phase $[14,15])$. Note that, in this case, there is no electrostatic restriction to keep the inplane polarization at the same value in the PTO and STO layers. Therefore, they are decoupled and tend to the corresponding bulk values. The magnitude of the rotations along the $z$ axis are strongly reduced and also tend to bulk, while the tiltings along the [110] axis become the dominant AFD mode. As before, the tiltings in the PTO-like octahedra are enhanced with respect to the bulk values due to the interfacial coupling with the STO-like octahedra.

Interestingly, at intermediate strains (around $\varepsilon \approx 0$ ) the polarization rotates continuously from the $c$ to the $a a$ phase ( $r$ phase $[14,15])$. Within this regime the electromechanical response of the system $\left(d_{31}\right.$ and $d_{11}$ piezoelectric constants) is enhanced, and at the STO lattice constant amounts to $0.30 \mathrm{nC} / \mathrm{N}$, twice as large as the $d_{33}$ in $\mathrm{Pb}\left(\mathrm{Zr}_{0.5} \mathrm{Ti}_{0.5}\right) \mathrm{O}_{3}$ at room temperature [17]. The appearance of low symmetry phases where the polarization is rotated away from the substrate normal has been already reported on PTO thin films grown on $\mathrm{DyScO}_{3}$ under tensile strains $(+1.4 \%)$ [18]. However, we do observe this rotation of polarization and the associated enhanced piezoelectric response for much smaller strains. This is due to the increased stability of the $r$ phase in this system, which is caused by the constrain imposed by the STO on the polarization of the PTO layer. Our simulations suggest that, in order to reduce $P_{z}$, PTO prefers a rotation keeping large the magnitude of $\mathbf{P}$ over a monotonic reduction, a well-known fact in FE perovskite oxides $[17,19]$.

As a summary of the coupling between strain and FE and AFD modes, we observe a rotation of the main axis of the system (defined by both the direction of the polarization and the rotation axis of the octahedra) from out of plane for compressive strains to in plane for tensile strains. For the AFD modes, this trend can be understood if we consider that the oxygen cage is very rigid. Then, as strains are applied, the system allows the $\mathrm{TiO}_{6}$ octahedra to reorient to maintain their shape (see Fig. 3 of Ref. [10]). More importantly, here we observe an extra interface coupling between the FE polarization direction and AFD modes that reveals itself in the different rotations of the octahedra types defined before. The largest difference is observed when strong compressive strains are applied: here the $\mathrm{P}^{+}$ octahedra rotate more than PTO and STO ones, while $\mathrm{P}^{-}$ octahedra rotate much less. In order to understand this result let us discuss the origin of the coupling between FE and AFD modes in this system.

AFD distortions are usually regarded as purely steric phenomena, where the rotation of the octahedra takes place if the $A$ ion is small enough to let the $B-\mathrm{O}-B$ bond bend [20]. A polar distortion of the $A$ cation along the positive $z$ axis reduces its distance with the oxygen ions of the $\mathrm{TiO}_{2}$ plane immediately above while it increases the distance with the ones below [Fig. 3(a)]. Taking into account that the ionic radii for $\mathrm{Sr}^{2+}$ and $\mathrm{Pb}^{2+}$ are almost the same (1.26 and $1.29 \AA$, respectively) and using a steric model, we would expect that a stronger rotation would only be favored if the mean oxygen- $A$ cation distance increases, leaving space for the octahedra to rotate. However, we find that the mean $A-\mathrm{O}$ distance is very similar for both $\mathrm{P}^{+}$and $\mathrm{P}^{-}$octahedra, close to $2.78 \AA$, while the rotations are very different. In order to explain this difference in rotations, we propose that the driving force in the mixed AFD-FE-strain coupling in PTO/STO superlattices is of covalent nature, instead of steric. It is well known that a chemically active lone pair on the $\mathrm{Pb}$ ion, which allows for strong covalent hybridization with $\mathrm{O}$, lies at the origin of FE in bulk PTO. For $\mathrm{P}^{+}$, due to the coupling between FE and AFD distortions, not all the $\mathrm{Pb}-\mathrm{O}$ bonds are equivalent. In particular, having a shorter $(2.45 \AA)$ and a longer one $(2.93 \AA)$ allows the $\mathrm{Pb}$ complex to acquire a pseudotetrahedral shape typical of many covalent $\mathrm{Pb}^{2+}$ compounds. For the $\mathrm{P}^{-}$octahedra, the polar distortion increases the $\mathrm{Pb}$ $\mathrm{O}$ distances and the previous mechanism does not apply [Fig. 3(b)]. These results agree with recent ab initio calculations that emphasize the role of covalent interactions in

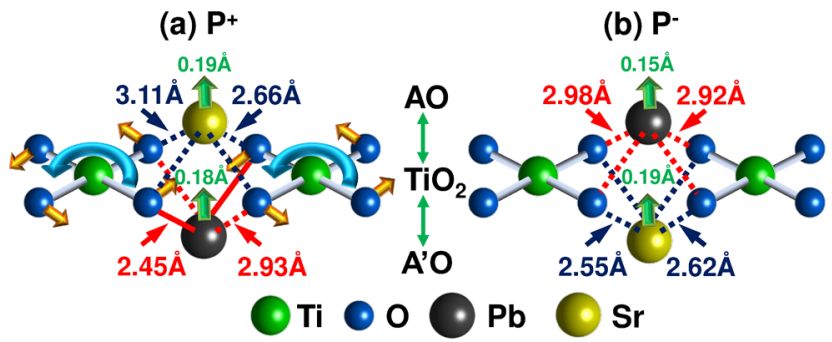

FIG. 3 (color online). (a) Diagram showing the change in distances between $\mathrm{Pb}$ and $\mathrm{Sr}$ ions with $\mathrm{O}$ anions at the PTO/STO interface under compressive strain for a $\mathrm{P}^{+} \mathrm{TiO}_{6}$ octahedra. (b) Same as in (a) but for a $\mathrm{P}^{-}$octahedra. Reduction of distance and reinforcement of the $\mathrm{Pb}-\mathrm{O}$ bond is shown by full red lines while an increase in the $\mathrm{Pb}-\mathrm{O}$ distance and weakening of the bond is shown by dotted lines. Blue dotted lines represent $\mathrm{Sr}-\mathrm{O}$ distances. Green arrows on $A$ cations $(\mathrm{Pb}$ or $\mathrm{Sr}$ ) represent out-of-plane displacement of the corresponding atoms, whose magnitude is written in green above the arrows. Yellow arrows on oxygen atoms represent their in-plane displacements. 
TABLE I. Polarization and relative energies of the different monodomain configurations for superlattices as a function of the periodicity of the supercell. In-plane strain corresponds to a STO substrate, with a theoretical in-plane lattice constant of $a_{\|}=3.874 \AA$. GS stands for ground state. Polarizations in $\mu \mathrm{C} / \mathrm{cm}^{2}$, energies in $\mathrm{meV}$ per five atom perovskite unit cell.

\begin{tabular}{lccccccccc}
\hline \hline & & $(1 / 1)$ & & & $(2 / 2)$ & \multicolumn{3}{c}{$(3 / 3)$} \\
& $P_{\text {STO }}$ & $P_{\text {PTO }}$ & $E$ & $P_{\text {STO }}$ & $P_{\text {PTO }}$ & $E$ & $P_{\text {STO }}$ & $P_{\text {РTO }}$ & $E$ \\
\hline Paraelectric & $(0,0,0)$ & $(0,0,0)$ & +15.3 & $(0,0,0)$ & $(0,0,0)$ & +12.8 & $(0,0,0)$ & $(0,0,0)$ & +9.7 \\
{$[110]$} & $(21,21,0)$ & $(31,31,0)$ & +4.6 & $(16,16,0)$ & $(35,35,0)$ & +3.5 & $(14,14,0)$ & $(36,36,0)$ & +3.5 \\
{$[001]$} & $(0,0,36)$ & $(0,0,35)$ & +1.2 & $(0,0,34)$ & $(0,0,35)$ & +3.1 & $(0,0,34)$ & $(0,0,34)$ & +4.0 \\
{$[111]$} & $(14,14,32)$ & $(23,23,31)$ & $\mathrm{GS}$ & $(9,9,30)$ & $(30,30,30)$ & $\mathrm{GS}$ & $(7,7,29)$ & $(32,32,30)$ & $\mathrm{GS}$ \\
\hline \hline
\end{tabular}

the origin of AFD distortions [21]. As the in-plane strain is increased, the polarization rotates away from the $z$ axis and this coupling is reduced making the in-plane rotations small and similar for all octahedra-types when the values of the strain are larger than $+1 \%$. Under these tensile strains, the $\mathrm{Pb}$ displaces in plane and both $\mathrm{P}^{+}$and $\mathrm{P}^{-}$ become equivalent.

We have also carried out simulations for different periodicities, but fixing $a_{\|}$to the LDA theoretical one of STO. We perform both unconstrained and constrained structural optimizations, where we impose a purely out-of-plane or in-plane polarization on the superlattice. Relative energies and polarizations of the PTO and STO layers are gathered in Table I, while the corresponding rotation angles can be found in the Supplemental Material [22]. The GS monodomain configuration displays both in-plane and out-ofplane polarizations, independently of $n$, although for $n=1$ the GS $r$ phase reported in Table I is essentially degenerated with the $c$ phase (the difference in energy, $1.2 \mathrm{meV}$ per 5 atom perovskite unit cell, is within the accuracy of our simulations). This delicate competition was already observed by Bousquet et al., where the phonon frequency of the mode involving in-plane distortions in the $(1 / 1)$ GS of Ref. [9] was found to be only $6 \mathrm{~cm}^{-1}$, close to becoming unstable. The small difference between the results in Table I and those in Ref. [9] can be ascribed to small changes in the methodology. Larger periodicities of the superlattice seem to increase the range of stability of the $r$ region, as the difference in energies between this phase and the rest increases. For $n \geq 2$, within the PTO layer, $\mathbf{P}$ lies close to the diagonal of the perovskite unit cell (configuration labeled as [111] in Table I). The GS can be considered as a condensation of $\mathrm{FE}_{z}+\mathrm{FE}_{x y}+\mathrm{AFD}_{z}+\mathrm{AFD}_{x y}$ modes. In every case, $P_{z}$ is nicely preserved at the $\mathrm{PTO} / \mathrm{STO}$ interface, with a value between 30 and $35 \mu \mathrm{C} / \mathrm{cm}^{2}$, in good agreement with previous first-principles simulations on clean $\mathrm{PbTiO}_{3} / \mathrm{SrTiO}_{3}$ interfaces [23].

The structures obtained from the previous relaxations serve also as the starting point to answer the question about the evolution of the energy gain due to FE-AFD coupling with the periodicity of the superlattice, For this analysis we have focused on the [001] phase described above, since for this structure the separation of atomic displacements into polar distortions and oxygen octahedra rotations is trivial.
Similar energy decompositions are expected for the GS [111] structure. The method to disentangle the different energy contributions due to the polar displacements, oxygen octahedra rotations, and/or their coupling can be found in the Supplemental Material [22]. The results are depicted in Fig. 4 as a function of the superlattice periodicity. It immediately follows that for ultrashort periodicities the energy contribution from the coupling terms is negative and very large, significantly increasing the stability of the polarized structure. This points in the direction of previous theoretical results [9] that support an "improper ferroelectricity" origin for the polarization in these ultrashort periodicities. However, the larger the periodicity, the smaller the importance of the coupling term, which even changes its sign for $n \geq 3$. From this point on, we can consider our superlattices to behave as normal ferroelectrics, in good agreement with experimental results [according to Ref. [7], $(3 / 3)$ was the threshold periodicity above which the normal FE behavior was recovered].

In summary, our first-principles simulations show how the FE-AFD-strain coupling in monodomain PTO/STO superlattices produces a phase diagram much richer than initially envisaged. The driving force of the coupling is a combination of electrostatic and covalent effects. The new phases might contribute to the stabilization of the monodomain configuration over the recently observed and competing polydomain structures [24]. The experimental

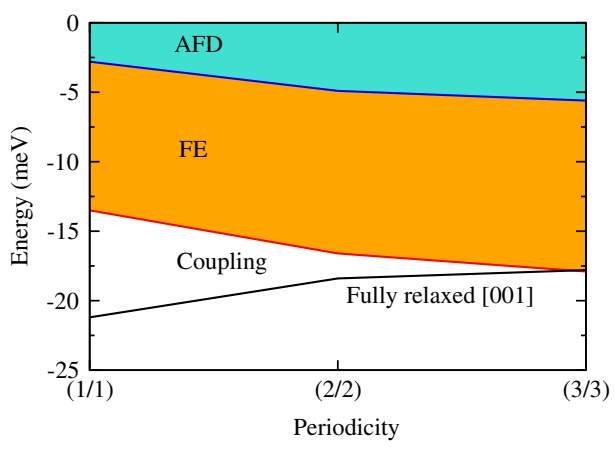

FIG. 4 (color online). Decomposition of the energy of the [001] phase into contributions from the polar modes, the AFD modes, and the coupling between them. The reference energy corresponds to a structure where neither polar nor AFD instabilities are allowed to condense. 
observation of the in-plane component of the polarization in the superlattices remains to be confirmed.

We acknowledge P. Zubko, Ph. Ghosez, and J.-M. Triscone for the critical reading of the manuscript. J. J. is indebted to Dr. J. de la Dehesa for useful discussions. We acknowledge financial support from Grants No. FIS200912721-C04-02 and No. CP-FP 228989-2 OxIDes. We acknowledge the computer resources, technical expertise, and assistance provided by the Red Española de Supercomputación. Calculations were also performed at the ATC group of the University of Cantabria.

[1] M. Dawber, K. M. Rabe, and J. F. Scott, Rev. Mod. Phys. 77, 1083 (2005).

[2] Ph. Ghosez and J. Junquera, Handbook of Theoretical and Computational Nanotechnology (American Scientific Publishers, Stevenson Ranch, CA, 2006) pp. 623-728.

[3] J. Junquera and Ph. Ghosez, J. Comput. Theor. Nanosci. 5, 2071 (2008).

[4] M. E. Lines and A. M. Glass, Principles and Applications of Ferroelectrics and Related Materials (Oxford University Press, Oxford, England, 1977).

[5] D. G. Schlom et al., Annu. Rev. Mater. Res. 37, 589 (2007).

[6] X. Wu, K. M. Rabe, and D. Vanderbilt, Phys. Rev. B 83, 020104(R) (2011).
[7] M. Dawber et al., Phys. Rev. Lett. 95, 177601 (2005).

[8] M. Dawber et al., Adv. Mater. 19, 4153 (2007).

[9] E. Bousquet et al., Nature (London) 452, 732 (2008).

[10] J. M. Rondinelli and N. A. Spaldin, Adv. Mater. 23, 3363 (2011).

[11] N. A. Benedek and C. J. Fennie, Phys. Rev. Lett. 106, 107204 (2011).

[12] J. M. Soler et al., J. Phys. Condens. Matter 14, 2745 (2002).

[13] J. Junquera, M. Zimmer, P. Ordejón, and Ph. Ghosez, Phys. Rev. B 67, 155327 (2003).

[14] N. A. Pertsev, A. G. Zembilgotov, and A. K. Tagantsev, Phys. Rev. Lett. 80, 1988 (1998).

[15] O. Diéguez et al., Phys. Rev. B 69, 212101 (2004).

[16] J. B. Neaton and K. M. Rabe, Appl. Phys. Lett. 82, 1586 (2003).

[17] L. Bellaiche, A. García, and D. Vanderbilt, Phys. Rev. Lett. 84, 5427 (2000).

[18] G. Catalan et al., Phys. Rev. Lett. 96, 127602 (2006).

[19] H. Fu and R. E. Cohen, Nature (London) 403, 281 (2000).

[20] P. Woodward, Acta Crystallogr. Sect. B 53, 44 (1997).

[21] P. Garcia-Fernandez, J. Aramburu, M. Barriuso, and M. Moreno, J. Phys. Chem. Lett. 1, 647 (2010).

[22] See Supplemental Material at http://link.aps.org/ supplemental/10.1103/PhysRevLett.107.217601 for further technical details and structural data.

[23] V. R. Cooper, K. Johnston, and K. M. Rabe, Phys. Rev. B 76, 020103(R) (2007).

[24] P. Zubko et al., Phys. Rev. Lett. 104, 187601 (2010). 